\title{
Training and Credentialing in NOTES (Natural Orifice Translumenal Endoscopic Surgery)
}

\author{
John R. Romanelli • David J. Desilets
}

Published online: 16 October 2013

(C) Springer Science + Business Media New York 2013

\begin{abstract}
NOTES (Natural Orifice Translumenal Endoscopic Surgery) remains a largely experimental technique with scattered early case reports and a series showing safe and efficacious implementation. Most of these reports revolve around transvaginal cholecystectomy, with rigid and flexible endoscopic instrumentation utilized. The conundrum of how to train surgeons in this evolving area remains a vexing problem. Animate labs remain the hallmark of training for new procedures but are expensive and require close contact with industry professionals to help supply the necessary equipment for NOTES procedures. Courses are held by societies and universities and can be a useful opportunity for training. Virtual reality simulators can be a potential avenue for skill acquisition, but these are also costly and early in development. Multidisciplinary teams of surgeons, gastroenterologists, and gynecologists remain a recommended concept in the development of a new NOTES program. While credentialing remains a local issue, Institutional Research Board approval should still be entertained before beginning NOTES procedures.
\end{abstract}

Keywords Natural orifice surgery - Training . Credentialing - Transvaginal access $\cdot$ Natural orifice translumenal endoscopic surgery (NOTES) · Endoscopy · Rigid endoscopy $\cdot$ Multidisciplinary team

\footnotetext{
J. R. Romanelli $(\square)$

Department of Surgery, S3659, Baystate Medical Center, Tufts University School of Medicine, 759 Chestnut Street, Springfield, MA 01199, USA

e-mail: john.romanelli@bhs.org
}

D. J. Desilets

Department of Medicine, Baystate Medical Center, Tufts University School of Medicine, Springfield, MA, USA

\section{Introduction}

As we enter the era of human NOTES (Natural Orifice Translumenal Endoscopic Surgery), it is unclear how we will implement natural-orifice surgical techniques. Certainly, the concept of fewer incisions and potentially less painful and faster recovery resonates with most surgeons. Indeed, early results of NOTES transvaginal cholecystectomy [1-3] demonstrate the feasibility and safety of the NOTES approach, but many barriers exist. These include cost, difficulty in performing these procedures, perception of increased risk, lack of critical instrumentation, and a lack of appropriate training.

One of the fundamental challenges to the adoption of NOTES as identified in the 2005 White Paper [4] is how to train future NOTES surgeons. At that time, the recommendation as laid out in this early seminal opinion paper, was that "training should occur as a team in a facility with good animal resources and equipment." We still believe this to be true. Further, in the White Paper, the recommendation was made that membership into the Natural Orifice Surgery Consortium for Assessment and Research (NOSCAR) required "a multidisciplinary team, such that the team possesses both advanced therapeutic endoscopic skills and advanced laparoscopic skills." While the adoption of early human NOTES cases has evolved away from multidisciplinary teams in some cases [5], we still believe this concept to be a best practice standard because it allows for capitalization on different skill sets. This will be discussed in detail below.

The conundrum of training and credentialing for NOTES procedures has multiple components. In exploring this issue we must first break apart the core tasks necessary to perform NOTES, and then seek to apply the concepts to the future training of surgeons interested in these procedures. 


\section{Surgeon as Endoscopist}

Flexible endoscopy, once the domain of the general surgeon, has largely been abandoned by many surgeons as a primarily surgical tool. While surgical trainees are required to have endoscopic experience in their residency training (with recent increased requirements for numbers), most would argue that their skill development is rudimentary and confined to basic endoscopic techniques. Some surgeons still perform percutaneous endoscopic gastrostomy (PEG) placement. Fewer still are proficient at endoscopic retrograde cholangiopancreatography (ERCP). Other skills such as endoscopic submucosal dissection (ESD) and endoscopic ultrasound (EUS) are rarer still among surgeons. Yet, all of the skills necessary to perform these techniques would be complementary to the skill set of the NOTES surgeon. Furthermore, the manipulation of the endoscope is largely confined to the hollow lumen; advanced endoscopic maneuvers outside the GI tract are necessary to reach the target anatomy, and may be beyond the skill level of a general surgeon endoscopist. Finally, many endoscopic tools routinely used by the interventional endoscopist are unfamiliar to the surgeon, thus limiting his ability to adapt endoscopic techniques to NOTES. For classically trained surgeons to become NOTES surgeons they must greatly improve upon the rudimentary endoscopic skill set developed during residency.

\section{Endoscopist as Surgeon}

Gastroenterologists/endoscopists, who might possess the requisite endoscopic skill set to perform NOTES procedures, nevertheless, are not routinely trained in surgical anatomy, basic laparoscopic and open surgical techniques, or principles of preoperative and postoperative patient care. They cannot convert to open or laparoscopic rescue should an intraoperative disaster occur, have not participated in surgical procedures except perhaps as medical students, and, in general, are poorly equipped to perform natural orifice surgery despite any endoscopic prowess they might possess. Furthermore, gastroenterologists do not generally have privileges in the operating room (OR), nor are they credentialed by their institutions to operate on patients. For classically trained gastroenterologists to become NOTES surgeons they must greatly improve upon the rudimentary surgical skill set developed during medical school or in any subsequent training. It is likely that it will require less additional training to make a general surgeon into a NOTES surgeon rather than to make an endoscopist into a NOTES surgeon. However, a multidisciplinary team could capitalize on the strengths and skill sets of both groups without lengthy additional training, and, indeed, this is the path that many groups have taken to begin performing NOTES.

\section{Endoscope as Surgical Platform}

Another factor to consider in training and credentialing of NOTES surgeons is the relative difficulty in maintaining a proper horizon. In laparoscopic surgery the horizon is kept in such a way as to keep the location of critical anatomic structures in their natural relation to one another; i.e., anterior is anterior on the video screen. However, because flexible endoscopy is typically confined to a hollow lumen, where the relative locations of anterior and posterior are unknown and rarely matter, the need to maintain a proper horizon is greatly diminished. In addition, torque maneuvers are commonly employed to reach intraluminal locations, which further obscure orientation. Thus, for a flexible endoscope to reach the gallbladder, the anatomic orientation of the anatomy may be different than the conventional laparoscopic view, which creates the potential for misidentification of anatomy with subsequent injury. While this may be a drawback for the use of the flexible endoscope in NOTES procedures, it is a further limitation for laparoscopic surgeons who are not accustomed to directionality being a less important component of the procedure. Further, while one might think that stabilization of the horizon would lead to an improved performance, a recent study [6] demonstrated exactly the opposite: artificial stabilization of the horizon on the image led to confusion and a decrement in performance. Also, triangulation of instruments is nearly impossible without the aid of additional laparoscopic ports and instrumentation (i.e., "hybrid NOTES" procedures), and all instruments passed through the scope exit the instrument channel in-line with the scope. This means that moving the instruments can only really be accomplished by moving the scope itself, which in turn changes the field of view. This is unnatural to most surgeons but familiar to an expert endoscopist, which again speaks to the need for a multidisciplinary team.

\section{NOTES with Rigid Endoscopy}

There is a developing trend in transvaginal surgery, and specifically with cholecystectomy, to use standard laparoscopic instrumentation and cameras via the vagina to perform the procedure $[5,7,8]$. Although this differs from the classic description of NOTES by omitting the use of the flexible endoscope [4, 9], commonly used surgical instruments can be employed. While simplifying some aspects of the procedure, issues such as internal retraction, transvaginal access, and navigating out of the pelvis can remain 
challenges that require training. This may also obviate the need for the aforementioned multidisciplinary team, which may reduce scheduling difficulties. In addition, credentialing for such procedures becomes a much simpler concept. Further, with the development of surgical instrumentation for single-site approaches, some of these can be used for transvaginal procedures, and concepts learned from single-site or single port laparoscopic operations can be applied to the performance of NOTES procedures with conventional surgical tools. This does not imply that there are not inherent training challenges that arise with this approach, but faster adoption by general surgeons or gynecologic surgeons familiar with standard instrumentation is more likely.

\section{Transvaginal Access}

Most general surgeons do not commonly perform vaginal surgery; as such, gynecologic colleagues have often collaborated with general surgeons to help learn the anatomy and safely access the abdominal cavity. This could create credentialing issues, which will be commented on later in this document. Certainly, there is risk to the patient that is unique to transvaginal access (e.g., rectal injury or dyspareunia is unheard of in a laparoscopic cholecystectomy), and this must be explicitly discussed during the informed consent process. Whether or not to continue collaboration with gynecologists or to proceed to independent functioning after a defined number of cases is a decision best handled locally. There is insufficient literature to support a fixed number of mentored procedures before a non-gynecologic surgeon may safely access the abdomen transvaginally. However, there is a forthcoming position statement from NOSCAR that states that transvaginal access to the abdominal cavity is no longer considered experimental. Not only is it hoped that this document can help improve the likelihood of reimbursement, but it may serve as a guide for credentialing bodies.

\section{Multidisciplinary Team}

The 2006 NOTES White Paper [4] discusses the use of a multidisciplinary team to perform NOTES procedures, namely surgeons and gastroenterologists with advanced therapeutic endoscopic skills. This approach exploits the strengths and helps overcome the weaknesses of each group, possibly leading to improved outcomes. The development of peroral endoscopic esophageal myotomy (POEM) further blurs the lines between surgeon and gastroenterologist, and is often still performed, at least in the US, by multidisciplinary teams. One drawback to the team approach is the expensive resource of multiple physicians working together (eschewing reimbursement, in certain situations). In addition, team training, whether in the animal lab, simulation, or in the human OR, can help to clarify definitive roles for each surgeon so that confusion over who is operating and who is assisting is minimized. We still recommend the use of a multidisciplinary team for both transvaginal cholecystectomy and POEM, but also recognize the limitations in scheduling, access to care, and reimbursement.

\section{Starting a NOTES Program: Training}

So, on the assumption that your hospital wanted to start a NOTES program, what elements would be necessary? First, identification of like-minded collaborators, who could share expertise and skill sets, is essential. We recommend at least one surgeon with advanced minimally invasive skills, and one gastroenterologist with advanced therapeutic endoscopic skills. Our experience as a team has taught us that surgeons can benefit from the endoscopist's technical skills and knowledge of endoscopic accessories and instrumentation. Conversely, endoscopists can similarly benefit from the surgeon's knowledge of instrumentation, extraluminal anatomy, and management of complications or unexpected findings should they occur. Over time, the cross-pollination of thought benefits both parties, such that the surgeon starts to think like an endoscopist, and vice versa.

We also strongly endorse the need for an on-site animal lab. At the current time, virtual reality simulators that can substitute the real challenges of performing surgery with endoscopic tools are under development, but none have become the sine qua non of NOTES training. One must master the navigation of the endoscope to the target anatomy, the creation of the access to the abdominal cavity be it transvaginal or transgastric, the performance of the operation itself, typically aided by the use of laparoscopic ports and instrumentation, and all the while paying heed to the realistic physiologic stress of a living animal. Also, the aforementioned challenge of viewing the surgical field through the endoscope with the directionality altered must become comfortable prior to embarking upon human NOTES cases.

Animal experimentation requires approval from the institution's animal care and use committee. It also requires a prior investment in the necessary endoscopic equipment, laparoscopic equipment, procurement of laboratory space, and training of staff to maintain such tools. The value of partnership with industry representatives should not be underestimated. This can serve the dual purpose of helping to equip the lab and train the surgeon, and also can lead to fertile ground for new applications or new ideas for device development by industry. In some cases tasks need only be practiced on animal organ explants only (e.g., gastrotomy 
closure). This is not only much less costly, but does not typically require animal care committee approval.

One new simulator, the endoscopic-laparoscopic interdisciplinary training entity (ELITE), has been shown to be helpful in performing NOTES cholecystectomy [10•] by reducing complications and shortening overall OR time in animal models. The same simulator has also shown both face and construct validity to a large group of surgeons in performing NOTES appendectomy in the model [11•]. Another attempt at NOTES simulation, called NOSsE (Natural Orifice Simulated surgical Environment), was developed out of a laparoscopic box trainer, although formal validity studies are lacking at the current time [12]. Finally, a group led by Suvranu De [13] at Rensselaer Polytechnic Institute has been awarded an NIH grant to develop a NOTES simulator, and their first model simulating transvaginal cholecystectomy with a rigid endoscope was demonstrated at the 8th International NOSCAR Summit in July, 2013. While not yet widely available, simulators such as these may become useful training adjuncts.

\section{Training Opportunities}

Another more traditional component of training is the attendance at CME courses on NOTES. Some of these have been given in the university setting; some are sponsored at annual meetings given by societies such as the American Society of Gastrointestinal Endoscopy (ASGE) or the Society of American Gastrointestinal and Endoscopic Surgeons (SAGES). Also, there may be industry-sponsored non-CME courses which can aid in the training process. Prior to embarking upon our first human NOTES cases, our team attended formal, didactic courses with on-site animal labs both in New York and in Strasbourg, France. We found these to be extraordinarily helpful, as they allowed us to tap into the expertise of other surgeons who had more experience and had suffered through some of the same learning curve as did we.

One other essential component prior to embarking upon human NOTES cases is to directly observe cases being done by more experienced surgeons. Our team was very fortunate to have traveled to Yokohama, Japan to observe five POEM cases before we performed our own first case. In fact, that experience combined with our animal lab experience and history of successful human NOTES work, led to approval to begin POEM by our Credentialing Committee.

\section{Credentialing}

The issue of how to credential surgeons for NOTES procedures is no different from other credentialing issues in that it is primarily a local issue. That is, hospitals will have different procedures and by-laws governing the introduction of new procedures into practice. Further, the 2006 White Paper [4] recommended that all NOTES procedures be done under Institutional Research Board (IRB) approval, and that recommendation has not yet changed. This recommendation may, therefore, introduce additional regulatory hurdles to be cleared for credentials to be granted. Of note, some hospitals in the United States are beginning to consider transvaginal access to the abdomen as established and not experimental,which may alter the route necessary to gain privileges to perform transvaginal procedures. Once again, a forthcoming position paper from NOSCAR on this topic may help to address this issue.

There remain several issues in the arena of credentialing that bear mention. Some hospitals have several bodies that govern the use of new technology or procedures, and approval may be needed from these additional committees in order to proceed. Typically, IRB approval is necessary to perform a new procedure, and starting a NOTES program in a surgeon's institution would qualify. A credentialing body would then examine the issue of how the surgeon or team was trained. As noted above, there are hospital-based courses, meeting-based courses, a VR simulator (that is not widely available), animal labs, instructional videos, and direct case observation, all of which can be used to support an application for NOTES privileges. Anatomic concerns that may govern the granting of privileges, such as a general surgeon performing transvaginal access, must be taken into consideration. Often expert opinion plays a key role (i.e., a gynecologist certifying that a surgeon can perform transvaginal access independently and safely after direct observation of several cases). Detailed discussion with the Chairman of the Department and/or the Medical Director of the Operating Room may be necessary, as they too would likely have to grant the credentials. Finally, financial considerations should be considered before embarking upon human NOTES cases. While not the direct purview of the credentials process, one may wish to explore the economic ramifications prior to requesting privileges. In the case of our group, we were forced to demonstrate fiscal responsibility prior to gaining IRB approval or privileges to perform transvaginal cholecystectomy.

\section{Conclusions}

Human NOTES cases have begun to emerge as alternate techniques to treat abdominal and thoracic disease. The issue of how to foster safe patient outcomes through proper training and credentialing continues to loom as a large barrier to the widespread adoption of NOTES. Multidisciplinary teams may speed the learning process and afford 
complementary skill sets to avoid complications and improve outcomes. Animal experience is highly recommended prior to beginning human NOTES cases. As the number of different types of procedures begins to grow, direct case observation may be helpful to gain insight into the technical difficulties and instrument needs of these procedures. While credentialing is a local issue for hospitals, precedent for the adoption of new technologies and introducing new procedures should be considered in the granting of privileges for NOTES surgeons.

\section{Compliance with Ethics Guidelines}

Conflict of Interest John R. Romanelli has served as a consultant for NovaTract and has received honoraria for proctoring from Lourdes Healthcare. David J. Desilets declares that he has no conflict of interest.

Human and Animal Rights and Informed Consent This article does not contain any studies with human or animal subjects performed by any of the authors.

\section{References}

Papers of importance, published recently, have been highlighted as:

- Of importance

1. Marescaux J, Dallemagne B, Perretta S, Wattiez A, Mutter D, Coumaros D. Surgery without scars: report of transluminal cholecystectomy in a human being. Arch Surg. 2007;142(9):823-6 discussion 826-7.

2. Zorron R, Maggioni LC, Pombo L, Oliveira AL, Carvalho GL, Filgueiras M. NOTES transvaginal cholecystectomy: preliminary clinical application. Surg Endosc. 2008;22(2):542-7 Epub 2007 Nov 20.

3. Gumbs AA, Fowler D, Milone L, Evanko JC, Ude AO, Stevens P, Bessler M. Transvaginal natural orifice translumenal endoscopic surgery cholecystectomy: early evolution of the technique. Ann Surg. 2009;249(6):908-12.
4. Rattner D, Kalloo A, ASGE/SAGES Working Group. ASGE/ SAGES Working Group on natural orifice translumenal endoscopic surgery, October 2005. Surg Endosc. 2006;20(2):3-329.

5. Roberts KE, Shetty S, Shariff AH, Silasi DA, Duffy AJ, Bell RL. Transvaginal NOTES hybrid cholecystectomy. Surg Innov. 2012;19(3):230-5.

6. Sodergren MH, Warren A, Nehme J, Clark J, Gillen S, Feussner H, Teare J, Darzi A, Yang GZ. Endoscopic horizon stabilization in natural orifice translumenal endoscopic surgery: a randomized controlled trial. Surg Innov. 2013. doi:10.1177/1553350613 489187.

7. Zornig C, Emmermann A, von Waldenfels HA, Mofid H. Laparoscopic cholecystectomy without visible scar: combined transvaginal and transumbilical approach. Endoscopy. 2007;39(10): 913-5.

8. Forgione A, Maggioni D, Sansonna F, Ferrari C, Di Lernia S, Citterio D, Magistro C, Frigerio L, Pugliese R. Transvaginal endoscopic cholecystectomy in human beings: preliminary results. J Laparoendosc Adv Surg Tech A. 2008;18(3):345-51.

9. Kalloo AN, Singh VK, Jagannath SB, Niiyama H, Hill SL, Vaughn CA, Magee CA, Kantsevoy SV. Flexible transgastric peritoneoscopy: a novel approach to diagnostic and therapeutic interventions. Gastrointest Endosc. 2004;60(1):114-7.

10. • Gillen S, Fiolka A, Kranzfelder M, Wolf P, Feith M, Schneider A, Meining A, Friess H, Feussner H. Training of a standardized natural orifice transluminal endoscopic surgery cholecystectomy using an ex vivo training unit. Endoscopy 2011;43(10):876-81; Epub 2011 Aug 10. This paper is important because it is the first report of an ex vivo training model for a NOTES procedure. This represents an important first step toward training for NOTES procedures.

11. • Gillen S, Gröne J, Knödgen F, Wolf P, Meyer M, Friess H, Buhr HJ, Ritz JP, Feussner H, Lehmann KS. Educational and training aspects of new surgical techniques: experience with the endoscopic-laparoscopic interdisciplinary training entity (ELITE) model in training for a natural orifice translumenal endoscopic surgery (NOTES) approach to appendectomy. Surg Endosc. 2012; 26(8):2376-82. This paper is also important, because it represents further development of the aforementioned simulator for NOTES procedures. In this paper, it was applied to appendectomy.

12. Clark J, Sodergren M, Noonan D, Darzi A, Yang GZ. The natural orifice simulated surgical environment (NOSsE): exploring the challenges of NOTES without the animal model. J Laparoendosc Adv Surg Tech A. 2009;19(2):211-4.

13. Suvranu De. Personal observation at demonstration, 8th International NOSCAR Summit, 19 July, 2013. 